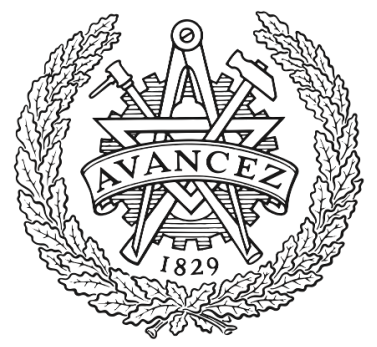

CHALMERS

UNIVERSITY OF TECHNOLOGY

\title{
Market-based Energy Management Model of a Building Microgrid Considering Battery Degradation
}

Downloaded from: https://research.chalmers.se, 2023-04-26 10:55 UTC

Citation for the original published paper (version of record):

Antoniadou-Plytaria, K., Steen, D., Le, A. et al (2021). Market-based Energy Management Model of a Building Microgrid Considering Battery Degradation. IEEE Transactions on Smart Grid, 12(2): 1794-1804. http://dx.doi.org/10.1109/TSG.2020.3037120

N.B. When citing this work, cite the original published paper.

(O2021 IEEE. Personal use of this material is permitted.

However, permission to reprint/republish this material for advertising or promotional purposes 


\title{
Market-based Energy Management Model of a Building Microgrid Considering Battery Degradation
}

\author{
Kyriaki Antoniadou-Plytaria, Graduate Student Member, IEEE, David Steen, Le Anh Tuan, Member, IEEE, \\ Ola Carlson, and Mohammad Ali Fotouhi Ghazvini, Member, IEEE
}

\begin{abstract}
This paper presents a model for energy management system of a building microgrid coupled with a battery energy storage. The model can be used to dispatch the battery as a flexible energy resource using a market-based setting. The battery is modeled considering battery degradation and reallife operation characteristics derived from measurements at a residential building. The performance of the model was evaluated first with simulations and integrated afterwards to an energy management system, which was demonstrated at a real residential building (HSB Living Lab) equipped with photovoltaic and battery storage systems. The simulation results showed that the building owner, and subsequently the residents, could benefit from the proposed model in reduced annual cost up to $3.1 \%$ under the considered pricing scheme. The demonstration results showed that dispatch under the measurement-based model could decrease the undelivered energy over the daily requested amount from the battery from $13.3 \%$ to $3.7 \%$. Thus, the proposed model, which couples the measurement-based dispatch with battery degradation, can lead to a more accurate estimation of the building operation cost and an improved overall performance of battery as a flexible resource in building microgrids.

Index Terms-Battery degradation, battery energy storage, building microgrid, energy management system, optimization.
\end{abstract}

\section{NOMENCLATURE}

Sets

$\mathcal{H}$

$\mathcal{I} / \mathcal{K}$

$\mathcal{P}$

Indices

$i / k$

$p$

$t$

\section{Parameters}

Set of time steps (simulation horizon)

Set of discharging/charging sample data

Set of sample points of the lifecycle loss function

Index for discharging/charging sample data

Index for lifecycle loss function sample point Index for time step

Manuscript received May 8, 2020; revised August 22, 2020; accepted October 24, 2020. This work was supported in part by the Swedish Energy Agency in the framework of the Joint Programming Initiative ERA-Net Smart Energy Systems Focus Initiative Smart Grids Plus, with support from the European Union's Horizon 2020 Research and Innovation Programme under Grant 646039 and Grant 775970, and in part by the European Union's Horizon 2020 Research and Innovation Programme under Grant 864048. Paper no. TSG-00703-2020. (Corresponding author: Kyriaki Antoniadou-Plytaria.)

Kyriaki Antoniadou-Plytaria, David Steen, Le Anh Tuan, Ola Carlson, and Mohammad Ali Fotouhi Ghazvini are with the Department of Electrical Engineering, Chalmers University of Technology, 41296 Gothenburg, Sweden (e-mail: \{kyriaki.antoniadou, ankur.srivastava, ali.fotouhi, david.steen, tuan.le, ola.carlson\}@chalmers.se).

Color versions of one or more figures in this article are available at https://doi.org/10.1109/TSG.2020.3037120. Digital Object Identifier 10.1109/TSG.2020.3037120
$P_{t}^{L}$

$P_{t}^{P V}$

SoE $E_{\max }$

SoE $E_{\text {min }}$

$\eta_{\text {ch }} / \eta_{\text {dis }}$

$P_{t}^{s p o t}$

$C_{i}$

$C_{e}$

$\Delta t$

$\kappa$

$E_{\max }$

$M$

$C^{B, 0}$

$C_{p p}$

$\eta$

$P^{\text {cap }}$

$P_{i}^{-} / P_{i}^{+}$

$P_{i}^{d i s} / P_{i}^{c h}$

$S o E_{i}^{d i s}$

$S o E_{k}^{c h}$

$B_{1}, B_{2}$

$I_{c}$

$T$

$\boldsymbol{H}$

$\widehat{\rho}_{p}$

$\mathrm{DoD}_{p}$ Variables

$p_{t}^{i m} / p_{t}^{e x}$

$p_{t}^{-} / p_{t}^{+}$

$p_{t}^{d i s} / p_{t}^{c h}$

$x_{t i} / y_{t k}$

$\operatorname{dod}_{t}$

soe $_{t}$

$q$

$r^{p}$

$c^{B}$

$\rho_{t}$

$\xi_{t p}$
Active load

Active power from solar generation

Upper state-of-energy limit of the battery

Lower state-of-energy limit of the battery

Charging/discharging efficiency of the battery

Spot price $[\$ / \mathrm{kWh}]$

Grid charge for energy transmission $[\$ / \mathrm{kWh}]$

Reimbursement fee paid to producers of smallscale generation $[\$ / \mathrm{kWh}]$

Time discretization step

Power to energy ratio

Installed battery capacity [kWh]

A large positive number

Purchase cost of battery [\$]

Power-based grid tariff [ $\$ / \mathrm{kW} / \mathrm{month}]$

Percentage of end-of-life retained capacity

Converter capacity limit $[\mathrm{kW}]$

Sample measurements of output/input power from/to the battery cells

Sample measurements of discharging/charging power to/from the grid

Sample measurements of battery energy storage state-of-energy during discharging

Sample measurements of battery energy storage state-of-energy during charging

Cycle aging coefficients

Average charging C-rate

Temperature [K]

Adjacency matrix

Sample points of lifecycle loss percentage

Sample points of depth-of-discharge

Imported/exported power from/to the grid Output/input power from/to the battery cells

Discharging/charging power to/from the grid

Positive variables indicating choice of discharging/charging samples $i / k$

Depth-of-discharge for the battery energy storage

State-of-energy of the battery energy storage

Battery capacity loss in \%

Cost of peak power drawn from the grid [\$]

Cost of cycle-based battery degradation in [\$]

Lifetime loss percentage associated with $D_{o} D_{t}$

Positive variable related to the choice of lifecycle 
loss function sample point $p$ Cycle aging cost dependent on $D_{o} D_{t}$ Binary variable used with adjacency constraints $z_{t}$

Binary variable indicating charging or discharging

\section{INTRODUCTION}

$\mathbf{T}$ HE NEED for dispatchable renewable-based energy sources (RES) has increased the focus on connecting storage units to energy systems. Energy storages support the penetration of RES by reducing the grid power fluctuations they cause and can offer many other services that benefit the grid operators (e.g., peak shaving, load leveling, frequency regulation [1]).

\section{A. Background and Motivation}

Energy storage also emerges as a critical resource for microgrid (MG) energy management [2] offering services such as increased self-consumption of RES-based generation and energy arbitrage [3], which benefit the MG owners. Following a decrease in the battery cost [4], the installation of behindthe-meter battery energy storages (BESs) has increased [3], signifying their value in reducing the building electricity cost.

Energy management systems (EMSs) designed for building microgrids (BMGs) apply energy scheduling solutions that enable the end-users to fully utilize the BES potential in reducing the energy cost [5], [6]. The accuracy of the BES model used by the BMG energy management system (BMG-EMS) can affect the reliability of the solution in terms of delivered energy and cost calculation. The link between BES dispatch and BES degradation is also essential, as it can be exploited to further reduce the operation cost of the BMG [5]. For efficient dispatch and accurate evaluation of the BES utilization, it is important to consider both real-life performance and lifetime degradation of the BES.

\section{B. State-of-the-Art}

1) Battery Degradation: Some studies present results on optimal BES dispatch considering BES degradation cost (e.g., [7]-[16]). A penalty is often used in the objective function in order to reduce BES stress, usually by avoiding deep cycle depths and/or high power rates [7]-[9]. Other works consider the impact of low state-of-charge (SoC) [10]-[13], while the simplest approach is to limit the number of cycles [14].

In [7], a mixed-integer non-linear programming model links the aging cost to the cycle depth and updates the BES capacity per time-step. The BES degradation in [8] is a function of the power rates, while authors in [9] link aging cost to both cycle depth and charge/discharge rates. However, the studies [7]-[9] do not consider the depth-of-discharge (DoD) of each cycle.

In contrast, the degradation cost in [10] is calculated using an approximation that links capacity loss with a weighted sum of SoC levels. Authors in [11] also consider SoC level and use Q-learning to approximate the non-convex cycle aging cost. The rainflow algorithm is employed in [12], where the authors prove convexity of the degradation function and use a subgradient algorithm to approximate the solution of optimal
BES dispatch. The loss of lifecycle as a function of DoD is also studied in [13], although the specific DoD related to each cycle is not considered. The authors propose a piecewise linearization of the lifecycle loss function, where the BES sizing of an under planning MG is decided based on the expected degradation associated with the maximum DoD of all cycles.

Unlike most studies, which neglect calendar aging, the authors in [15] incorporate both calendar and cycle aging into a mixed-integer linear programming (MILP) problem considering their dependencies on time elapsed and cumulative throughput, respectively. However, a predefined desired BES lifetime must be entered as a parameter to include calendar aging in the MILP problem, while the impact of SoC is not evaluated in either cycle or calendar aging.

2) BES Scheduling Models: Up until now, studies on optimal BES dispatch that consider degradation use technical BES models, which are built on some simplifying assumptions regarding the BES operation, e.g., the BES power and efficiencies are assumed independent of SoC. The BES scheduling in [16], which is formulated as a Markov decision process, considers both degradation and effective charging/discharging power dependent on the SoC resulting in an improved BES model compared to previously mentioned works. Still though, the round-trip efficiency is considered to be constant.

A few recent works proposed models that can integrate the actual, non-linear behavior of a real BES in linear programming (LP) optimization problems [17]-[18]. The authors in [17] provide a piecewise linear approximation of the charging curve to account for the non-constant charging power limits, while simplifications are still applied on BES efficiency. In [18], each state of the BES operation is a linear combination of sampled points of operation taken from measurements. This approach considers dependency of both power and efficiency on SoC. Ref. [17]-[18] did not consider BES degradation.

\section{Paper Contributions and Structure}

This paper presents a market-based energy management model, which can be integrated in a BMG-EMS that uses BES as a flexible resource.

The BES is modeled using the sampling-based approach introduced in [17]-[18] to capture a more realistic BES operation performance. The proposed model also incorporates BES degradation.

The main contributions of this paper include the following:

- Development of a BMG energy management model with a market-based approach for BES dispatch. The model can be utilized by building owners for real-time (5$15 \mathrm{~min}$ ) energy management and monthly or annual assessment of the building energy cost. A measurementbased BES model and the impact of degradation are considered in the BES dispatch, while the effect of DoD is assessed both in cycle and calendar aging.

- Comprehensive evaluation of the BES dispatch under different technical and degradation BES models that reveals which modeling approach can yield the maximum reduction to the electricity cost of the residents. 
- Validation of the performance of the measurement-based BES model against the real behavior of an on-site BES in a demonstration site. The validation proves the effectiveness of integrating a measurement-based model in a BMG-EMS and confirms its advantage against conventional BES scheduling models.

The rest of the paper is organized as follows. Section II presents the model and Section III describes the study approach. Section IV discusses simulation results and Section $\mathrm{V}$ presents test results from a real demonstration site. Finally, Section VI presents the main conclusions from the paper.

\section{BMG EnERgy MANagement Model}

The proposed model is integrated in a BMG-EMS, which controls the BES using the forecasted values of the electricity price, photovoltaic (PV) generation and building electricity consumption as inputs. The model also uses the last measured value of the BES's state-of-energy (SoE) as an input, while the output is the BES charging/discharging power for each time step of the scheduling period. The BES throughput is calculated in Wh, and thus the term SoE is used instead of SoC [17]. However, the term SoC is still used for the experimental measurements of the BES. In addition, DoD is defined as the discharged energy from $100 \%$ SoE, i.e., $D o D=1-S o E$.

It is assumed that the building owner is also the BES owner as well as the BMG operator, and employs the BMG-EMS for economic BES dispatch considering a business model, where the BMG can purchase and sell energy to a retail electricity provider at wholesale market price. Moreover, it is assumed that the building owner intends to use the whole BES capacity that is available by the BES's operation limits in order to reduce the energy cost using energy arbitrage (load-shifting).

\section{A. Objective Function}

The BMG-EMS seeks to minimize the total cost given by:

$$
\begin{aligned}
& \min \sum_{t \in \mathcal{H}}\left(\left(P_{t}^{s p o t}+C_{i}\right) p_{t}^{i m} \Delta t-\left(P_{t}^{s p o t}+C_{e}\right) p_{t}^{e x} \Delta t\right) \\
& +r^{p}+c^{B} .
\end{aligned}
$$

The scheduling horizon and the time intervals are respectively shown by $\mathcal{H}$ and $\Delta t$ in (1). The positive variables $p_{t}^{i m} / p_{t}^{e x}$ are the imported/exported power from/to the grid at time step $t$. The first term in (1) is the cost of the imported energy, where $P_{t}^{\text {spot }}$ is the spot price and $C_{i}$ is the grid charge for energy transmission (grid utilization). The second term is the revenue associated with the energy exported to the grid. The reimbursement fee $C_{e}$ is paid by the distribution system operator (DSO) as an incentive to reduce network losses. The term $c^{B}$ denotes the cost of BES capacity loss due to cycle aging and $r^{p}$ is the cost for the peak power drawn from the main grid, which satisfies:

$$
r^{p} \geq C_{p p} p_{t}^{i m}, \forall t \in \mathcal{H}
$$

The power-based grid tariff $C_{p p}$ is linked to the maximum average power of the studied period (measured per $\Delta t$ ).

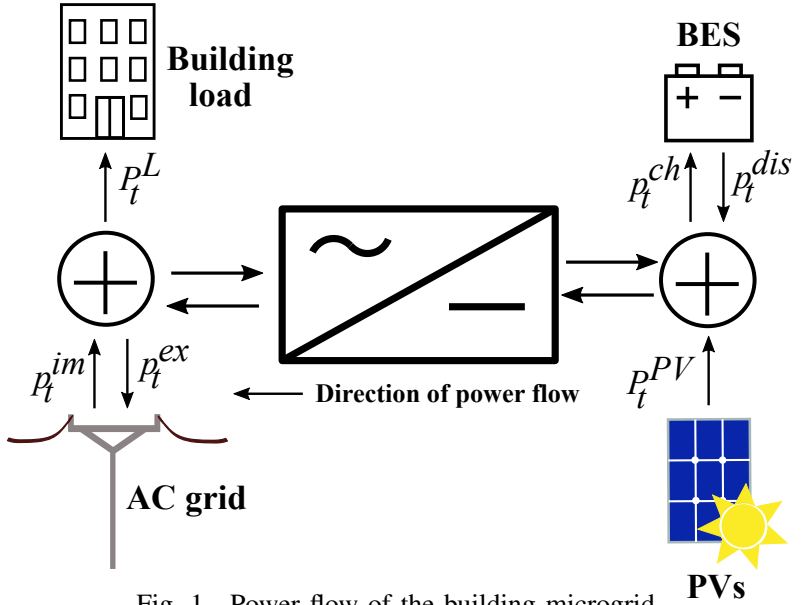

Fig. 1. Power flow of the building microgrid.

\section{B. Power Balance}

The PV system and the BES are connected to the upstream AC grid via a converter with bi-directional operation, since the solar energy and the BES stored energy can be exported to the AC grid and, in addition, the BES can be charged through both the upstream AC grid and the PV system (see Fig. 1). The power balance of the BMG is given by

$$
P_{t}^{P V}+p_{t}^{d i s}-p_{t}^{c h}=p_{t}^{e x}-p_{t}^{i m}+P_{t}^{L}, \forall t \in \mathcal{H},
$$

where $P_{t}^{P V}, P_{t}^{L}$, and the positive variables $p_{t}^{c h} / p_{t}^{d i s}$ respectively refer to $\mathrm{PV}$ generation, building electric power consumption, and charging/discharging power that the BES draws from the grid and the PVs or injects to the grid. The losses of the grid side converter are ignored. The PV generation and the discharging/charging power of the BES might be curtailed due to inverter capacity limitations:

$$
\left|P_{t}^{P V}+p_{t}^{d i s}-p_{t}^{c h}\right| \leq P^{c a p}, \forall t \in \mathcal{H} .
$$

\section{BES Scheduling}

1) Conventional Model: The BES model that has been most frequently used in the latest literature on BES scheduling (e.g., [7], [14], [19]-[20]) assumes that the SoE of the BES at each time step is linearly dependent on the cumulative BES throughput of the previous time steps. The charging/discharging energy efficiency and the power limits are considered to be constant and independent of the SoE level. This model is described by the following equations:

$$
\begin{gathered}
\text { soe }_{t}=\text { soe }_{t-1}+\eta_{c h} \frac{p_{t}^{c h} \Delta t}{E_{\text {max }}}-\frac{p_{t}^{\text {dis }} \Delta t}{\eta_{\text {dis }} E_{\text {max }}}, \forall t \in \mathcal{H} \\
S o E_{\text {min }} \leq \text { soe }_{t} \leq S o E_{\text {max }}, \forall t \in \mathcal{H}, \\
0 \leq p_{t}^{c h} \leq \kappa E_{\text {max }}, \forall t \in \mathcal{H}, \\
0 \leq p_{t}^{d i s} \leq \kappa E_{\text {max }}, \forall t \in \mathcal{H}, \\
p_{t}^{c h} \leq z_{t} M, \forall t \in \mathcal{H},
\end{gathered}
$$




$$
p_{t}^{d i s} \leq\left(1-z_{t}\right) M, \forall t \in \mathcal{H} .
$$

In the above formulation, $\kappa$ denotes the power to energy ratio, which determines the maximum charging/discharging power according to the specifications of the BES manufacturer.

2) Measurement-based Model: The assumptions that simplify the BES operation, i.e., the constant charging/discharging efficiencies and maximum power limits can be seen in (5), (7)-(8). Depending on the application and the BES's stateof-health (SoH), these assumptions may lead to mismatches between the estimated and actual SoE or the BES may fail to deliver the rated power. Therefore, a sampling-based approach, which was first presented in [18], is used to derive a model that captures more accurately the behavior of an actual BES by utilizing underlying patterns that exist in the charging/discharging data. This measurement-based model uses sample data from charging/discharging curves and satisfies (11)-(18), where the positive variables $p_{t}^{-} / p_{t}^{+}$represent power output/input from/to the BES cells respectively, before/after BES losses have been taken into account:

$$
\begin{gathered}
\text { soe }_{t}=\text { soe }_{t-1}+\frac{p_{t}^{+} \Delta t}{E_{\max }}-\frac{p_{t}^{-} \Delta t}{E_{\max }}, \forall t \in \mathcal{H}, \\
p_{t}^{-}=\sum_{i \in \mathcal{I}} P_{i}^{-} x_{t i}, \forall t \in \mathcal{H}, \\
p_{t}^{d i s}=\sum_{i \in \mathcal{I}} P_{i}^{d i s} x_{t i}, \forall t \in \mathcal{H}, \\
p_{t}^{+}=\sum_{k \in \mathcal{K}} P_{k}^{+} y_{t k}, \forall t \in \mathcal{H}, \\
p_{t}^{c h}=\sum_{k \in \mathcal{K}} P_{k}^{c h} y_{t k}, \forall t \in \mathcal{H}, \\
\text { soe }_{t}=\sum_{i \in \mathcal{I}} S_{o E_{i}^{d i s}} x_{t i}+\sum_{k \in \mathcal{K}} S o E_{k}^{c h} y_{t k}, \forall t \in \mathcal{H}, \\
\sum_{i}^{I} x_{i, t}=1,0 \leq x_{i, t} \leq 1, \forall t \in \mathcal{H}, \\
\sum_{k}^{K} y_{k, t}=1,0 \leq y_{k, t} \leq 1, \forall t \in \mathcal{H} .
\end{gathered}
$$

Unlike the conventional model, the dependency of maximum charging/discharging rates on SoE levels is considered in the measurement-based model. That is because the feasibility regions of BES power and SoE in the measurement-based model are not independent from each other. Instead, a 3-dimensional feasibility region is defined by (12)-(13) and (16)-(17) using the discharging sample data $\left(S o E_{i}^{d i s}, P_{i}^{-}, P_{i}^{d i s}\right)$ to constrain the values of the variables $\left(s_{t}, p_{t}^{-}, p_{t}^{d i s}\right)$, which are dependent to each other, during discharging. Similarly, (14)-(16) and (18) define the feasibility region of the variables $\left(s_{0}, p_{t}^{+}, p_{t}^{c h}\right)$ during charging using the charging sample data $\left(S o E_{k}^{c h}, P_{k}^{+}, P_{k}^{c h}\right)$. In other words, the sample data form the convex hull of the feasible region for BES operation points. Thus, the variables $\left(\right.$ soe $\left._{t}, p_{t}^{+}, p_{t}^{c h}, p_{t}^{-}, p_{t}^{d i s}\right)$ can be written as convex combinations of the sample measurements. Each convex combination (BES operation point) depends on the variables $x_{i, t}$ and $y_{k, t}$, which are associated respectively with the choice of discharging or charging sample data.

The charging sample data can be obtained by any charging curve $p_{t}^{c h}\left(\right.$ soe $\left._{t}\right)$ during a charging period for the BES. The samples $\left(S o E_{k}^{c h}, P_{k}^{c h}\right)$ can be directly taken from the curve, while to derive the corresponding value for $P_{k}^{+}$, one can solve (11) for $p_{t}^{+}$, where $s o e_{t}=S o E_{k}^{c h}$ and $\Delta t$ is equal to the time interval between this sample and the previously measured SoE value. The discharging sample data can be obtained in a similar way. The sample data should also include the BES state, where the BES is an open-circuit and fully charged or fully discharged according to [18].

This model also incorporates the variable (with respect to BES power and SoE) charging/discharging efficiencies of the BES system (both internal BES losses and DC/DC converter losses are considered), which are given by $\eta_{t}^{c h}=p_{t}^{+} / p_{t}^{c h}$ and $\eta^{d i s}=p_{t}^{\text {dis }} / p_{t}^{-}$, respectively, $\forall t \in \mathcal{H}$ [18]. As the efficiencies are correlated with the model's decision variables, the efficiency values (at each BES power and SoE level) depend on the feasible values of $x_{i, t}$ and $y_{k, t}$, which determine the choice of $p_{t}^{d i s}$ and $p_{t}^{-}$(or $p_{t}^{c h}$ and $p_{t}^{+}$during charging).

\section{BES Degradation}

The BES degradation can be expressed as loss of available capacity or increase in the BES resistance and is non-linearly linked to many factors [9] depending on the chemistry [21]. Cycle aging can increase due to high $\mathrm{C}$-rates, frequent cycling with high DoD, high operating temperatures, and operation in very high/low storage voltages (SoC levels). Calendar aging is more severe at high temperatures and SoC [21], [22].

1) Dependency of Cycle Aging on Cumulative Throughput: The degradation model presented in [23] and modified in [15], [24] is used to model the dependency of cycle aging on cumulative throughput:

$$
q=B_{1} e^{B_{2} I_{c}} \sum_{t \in \mathcal{H}}\left(p_{t}^{-}+p_{t}^{+}\right) \Delta t .
$$

In (19), $q$ represents the BES capacity loss in $\%$, while the pre-exponential and exponential factors $B_{1}$ and $B_{2}$ can be obtained from empirical fitting of experimental data. The daily average C-rate $I_{c}$ is entered as a parameter and thus (19) becomes linear. The BES cost used in (1) is calculated as:

$$
c^{B}=\frac{C^{B, 0} q}{100 \%-\eta},
$$

where $C^{B, 0}$ is the installation cost of the BES and $\eta$ is the end-of-life retained capacity percentage.

2) Dependency of Cycle Aging on DoD: The model presented in [25] is used to study the dependency of cycle aging on maximum DoD. Given a function of lifecycle percentage loss for one cycle of a specific DoD, the cycle-based BES degradation cost entered in the objective function is given by

$$
c^{B}=\sum_{t \in \mathcal{H}} c_{t}^{D o D},
$$




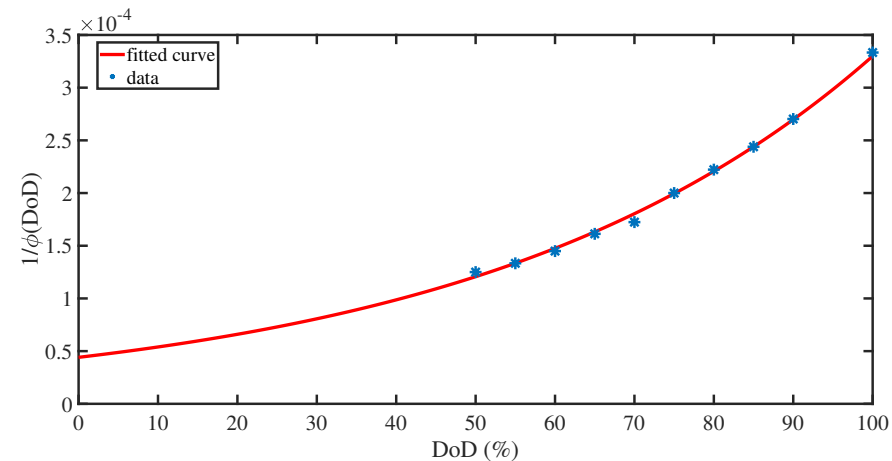

Fig. 2. The percentage of lifetime loss of one cycle at a specific DoD.

where $c_{t}^{D o D}$ is the cycle aging cost per time step $t$, given by:

$$
c_{t}^{D o D}=\max \left\{0,\left(\rho_{t}-\rho_{t-1}\right) C^{B, 0}\right\} .
$$

This cost is related to the percentage of lifetime loss $\rho_{t}=$ $1 / \phi(D o D)$ for a cycle at a specific DoD. An example of the loss of lifetime as a function of DoD derived from data of a Li-ion BES can be seen in Fig. 2 (based on data from [13]).

In (22), $\rho_{t}$ is greater than $\rho_{t-1}$ only when the BES is discharging, since the loss of BES lifetime increases, when the DoD increases. Thus, the aging cost is added for every discharging half-cycle, while it is zero during charging. As can be seen from (21)-(22), it is the starting DoD at the beginning of each discharging half-cycle and the $\mathrm{DoD}$ at the end of the respective half-cycle that increases the aging cost function. This formulation does not directly impact the charging or discharging time except for the purpose of limiting the number of discharging half-cycles within the time horizon. Two discharging half-cycles with the same starting and end DoD will induce the same aging cost even if the discharging time (number of time steps) is different between these two cycles. The degradation model can be piecewise linearized according to (23)-(27):

$$
\begin{gathered}
d o d_{t}=\sum_{p \in \mathcal{P}} D O D_{p} \xi_{t p}, \\
\rho_{t}=\sum_{p \in \mathcal{P}} \widehat{\rho}_{p} \xi_{t p}, \\
\sum_{p}^{P} \xi_{t p}=1,0 \leq \xi_{t p} \leq 1, \\
{\left[\begin{array}{c}
\xi_{t 1} \\
\xi_{t 2} \\
\vdots \\
\xi_{t P}
\end{array}\right] \leq[\boldsymbol{H}]\left[\begin{array}{c}
b_{1, t} \\
b_{2, t} \\
\vdots \\
b_{P-1, t}
\end{array}\right],} \\
\sum_{p=1}^{P-1} b_{p, t}=1 .
\end{gathered}
$$

In (23)-(24), $D O D_{p}$ and $\widehat{\rho}_{p}$ respectively refer to the sample points of DoD $\left(d o d_{t}\right)$ and lifetime loss percentage $\left(\rho_{t}\right)$, which are used for the piecewise linearization of the lifecycle loss function curve (Fig. 2). Eq. (26)-(27) are adjacency constraints, which are used to ensure interpolation of the decision variables $d o d_{t}$ and $\rho_{t}$ between consecutive sample points.

\section{E. Optimization Models}

Four optimization models are defined based on the employed BES scheduling and degradation models:

- Model-1: The conventional BES scheduling model (Section II-C1) is used, while degradation cost is neglected, i.e., the term $c^{B}$ is omitted from (1). The formulated MILP problem is given by (1)-(10).

- Model-2: A measurement-based BES scheduling model (Section II-C2) is used, which was validated with experimental values. As in Model-1, aging is neglected. The formulated LP problem is given by (1)-(4), (6), (11)-(18).

- Model-3: The measurement-based model is combined with the cycle aging model of Section II-D1. The formulated LP problem is given by (1)-(4), (6), (11)-(20).

- Model-4: The measurement-based model is combined with the cycle aging model presented in Section II-D2. The formulated MILP problem is given by (1)-(4), (6), (11)-(18), (21)-(27).

The optimization models were implemented in GAMS [26] interfacing CPLEX [27] to solve the LP and MILP problems.

\section{STUdy APPROACH}

A flow diagram of the approach adopted in the simulation studies and demonstrations of BES dispatch is depicted in Fig. 3. The four models presented in Section II-E have been applied in the simulation studies using an existing $7.2 \mathrm{kWh}$ BES with $6 \mathrm{~kW}$ charging/discharging power limits. The BES is installed at the HSB Living Lab (HSB LL) building [28], [29], located at the campus of Chalmers University of Technology. The BES dispatch under Model-1 and Model-2 was demonstrated at the same building to prove the increased accuracy of the measurement-based BES model compared to the conventional model and thus justify its integration in Model-3 and Model-4.

Nord Pool spot market prices [30] were used in the study, while the energy and power grid tariffs as well as the reimbursement fee were taken from the website of the local DSO [31]. For the peak power cost, the power tariff for commercial customers was used and scaled down to a cost that suited the scheduling time horizon (24h), as this is in practice a monthly fee paid to the DSO. The parameters for the measurementbased model were obtained from sample data of tests on the HSB LL BES, while $\eta_{c h}=0.91$ and $\eta_{d c h}=0.98$ for Model-1, corresponding to the average values that were recorded during those tests. The Li-ion cycle aging parameters of Model-3 were taken from [23] (where $B_{1}=0.0013$ and $B_{2}=0.3534$ ), while the BES loss function $\rho_{t}$, which is used in Model-4, was derived from Li-ion BES data provided in [13]. For the parameter $I_{c}$ in (19), which is included in Model-3, the daily average $\mathrm{C}$-rate was used, which was found to be 0.3 for the charging/discharging patterns of all four models over the 365 day-ahead simulations. 


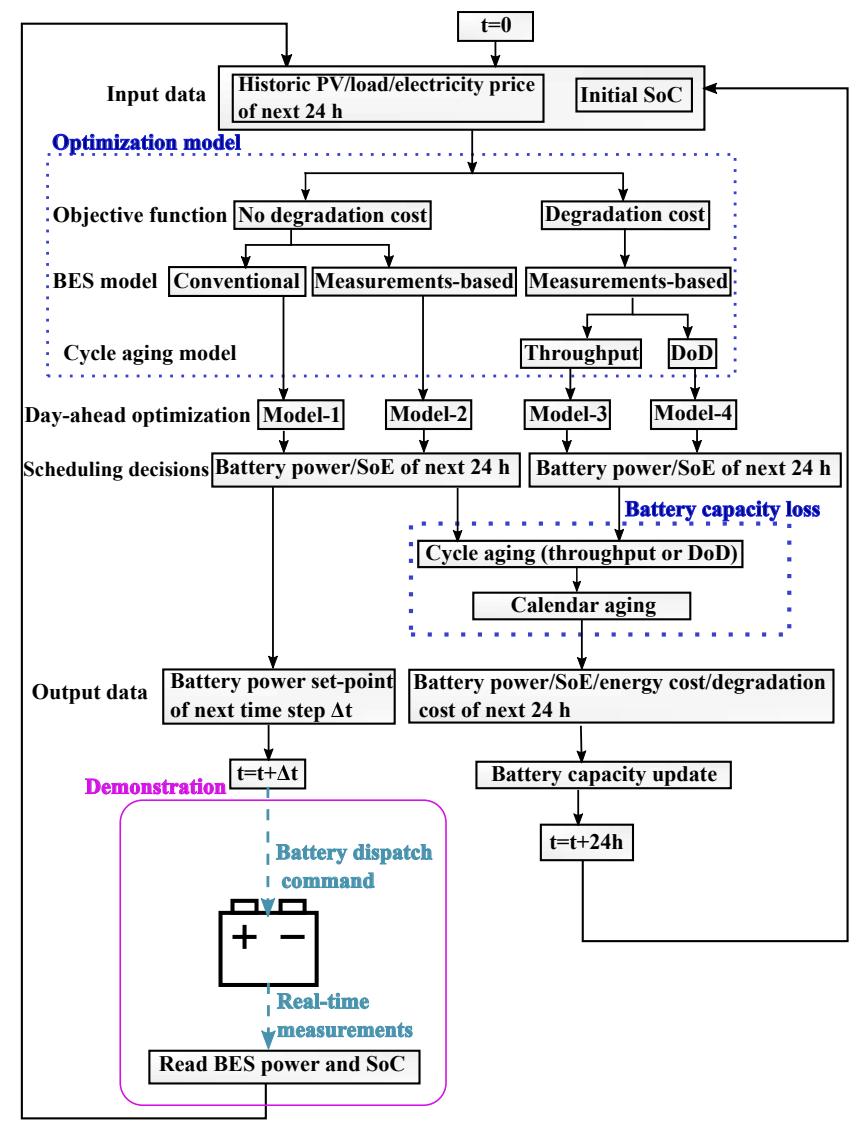

Fig. 3. The flow diagram presenting the simulation and demonstration of the BMG energy management model.

The main purpose of the simulations was to show the impact of different BES dispatch models on the building cost and degradation, while the purpose of the demonstration was to validate the accuracy of the BES scheduling models. Therefore, historical data of building consumption, PV generation, and electricity price were used as the input values to the models both in simulations and in demonstrations. These values are assumed to have been obtained by perfect forecasts.

A number of methodologies have been developed in the literature to forecast the short-term load demand, power generation and the electricity price [32]. It is essential to consider the complexity of each forecast tool, their strengths and weaknesses while choosing for the integration with an EMS. A review of various methodologies for threshold, probabilistic and point forecasting of the short-term electricity price is presented in [33]. The key techniques for short-term power forecast are statistic parametric methods, non-parametric intelligent methods and hybrid models, which are reviewed in [32]. Although the development of the forecast algorithms was outside of the scope of this paper, each of these techniques could be used to integrate with the proposed BMG energy management model.

\section{A. Simulations Studies}

The building load, PV generation, and electricity price data in 2018 were used to run 365 day-ahead BES scheduling simulations, where $\Delta t=5 \mathrm{~min}$. Considering the uncertainty in the future price of residential, stationary BESs [3], a sensitivity analysis was performed with three prices, i.e., \$100, \$290, and $\$ 500$ per $\mathrm{kWh}$, for the BES installation cost. These approximately correspond to a best-case, likely, and worstcase scenario. Moreover, two scenarios of SoE limits were investigated: $30 \%-80 \%$ (Scenario-1) and 10\%-90\% (Scenario$2)$. The initial and end SoE of each simulation was set to $50 \%$.

As shown in Fig. 3, the BES capacity was updated after each day-ahead optimization. The capacity loss was calculated again after the performed optimization to obtain the most accurate results, as the linearizations of the aging models introduced an average error of $4.6 \%$ and $14 \%$ to the cycle aging estimated by Model-3 and Model-4, respectively. First, the capacity loss due to cycle aging given by $c^{B}(100 \%-\eta) / C^{B, 0}$ was calculated using one of the models presented in Section II-D (dependency on throughput or DoD), where the real-time C-rate was used in (19). Afterwards, this loss was subtracted from the capacity that the BES had at the beginning of the day. Finally, the loss due to calendar aging was calculated according to (28) and subtracted from the remaining capacity for every $t$ that was an open-circuit period for the BES:

$$
Q_{r}=Q_{r, 0}-a_{c} \Delta t
$$

In (28), $Q_{r}$ is the percentage of retained capacity after the rest period, $Q_{r, 0}$ is the capacity percentage at the beginning of the rest period, and $a_{c}$ is a parameter dependent on SoC. Thus, the dependency of calendar aging on both SoC and rest period was considered. The values for $a_{c}$ were derived from linear interpolation of the calendar aging data given in [22].

The temperature in stationary BESs can easily be controlled and was therefore considered constant $(T=298 K)$. The BES of this study is installed in an indoor cabinet and according to the IEEE/ASHRAE standard [34], [35], it is the thermal management of the room rather than the individual BESs that controls the temperature of the stationary BESs. According to this standard, the supply air of the ventilation unit should assist in controlling the room temperature. Ideally, the optimization problem could seek to minimize the building energy cost by controlling the aggregated BES charging/discharging power and power consumption of the BES thermal management system. However, considering that the temperature of the room can be controlled during many months of the year through the heating system of the building and considering also that the power consumption of the BES thermal management system is relatively small in comparison with the BES power, then the impact of this power consumption to the total building cost can be considered negligible.

\section{B. Demonstrations}

A rolling horizon $(\mathrm{RH})$ approach was used for the demonstrations. The optimization problem was solved before each 15-minute time interval $(\Delta t)$. The set-point for the first time step was dispatched after each simulation, while the time horizon was shifted forward by $\Delta t$ for the next simulation, as shown in the flow diagram (Fig. 3). Since the RH approach was 
used and the set-points were continuously adjusted, Eq. (1) did not refer to the actual cost during the scheduling horizon.

The input values for load, PV generation, and spot price were updated at each time step to consider values that were outside of the previous simulation horizon (as the new simulation horizon had been shifted forward). The same input data (including initial SoC measured at $t=0$ ) were used in both demonstrations to allow for comparison of their results, while the SoE limits were set to $30 \%-80 \%$ (Scenario-1). If actual forecasts are to be used, then the updated input at each time step can also consider potential changes/updates in the forecasts. Thus, the rolling horizon approach can substantially reduce the impact of forecast and $\mathrm{SoE}$ estimation errors to the building cost by both renewing the input values and dynamically adjusting the set-points at each time step (the latter was implemented in the demonstrations).

\section{Simulation Results}

Fig. 4 presents the annual assessment metrics of the BES dispatch according to the four models presented in Section II-E in the two scenarios of SoE limits as described in Section III-A. The calculation of cycle aging and the respective cost is presented for both approaches (dependency on throughput/DoD) for comparison. The assessment of the models was performed according to the measurement-based BES model. Thus, the maximum feasible charging/discharging power was chosen, when the BES power set-points of Model-1 were infeasible with respect to the measurement-based model.

\section{A. Analysis}

1) Scenario-1: As Fig. 4(a) shows, Model-3 and Model-4 yielded the most economic operation at all prices. The total cost for either model was not higher than $\$ 4401$ at $\$ 100$, while Model-4 yielded the lowest cost at $\$ 290$ and $\$ 500$, which was not higher than $\$ 4591$ and $\$ 4795$, respectively. Even though the energy cost was increased in these models, the total capacity loss was lower than in Model-1 and Model2 , which did not include the cycle aging cost in their objective functions and gave a total cost of at least $\$ 4408$ at $\$ 100, \$ 4616$ at $\$ 290$, and $\$ 4847$ at $\$ 500$. Comparing with the highest cost of either Model-1 or Model-2, a reduction of up to $0.4 \%, 0.6 \%$, and $1.2 \%$ could be observed for Model-3 at the price of $\$ 100$, $\$ 290$, and $\$ 500$, respectively. Similarly, a reduction of up to $0.4 \%, 1.0 \%$, and $1.8 \%$ could be observed for Model-4 at the price of $\$ 100, \$ 290$, and $\$ 500$, respectively.

As the BES prices increase, the cycle aging decreases for Model-3 and Model-4. At the same time, calendar aging increases, as the BES is cycled less. Model-4 caused the lowest capacity loss among all models, which was not higher than $2.8 \%$ (at \$100). Interestingly, Model-2 caused the highest capacity loss due to cycle aging (at least $1.5 \%$ ), which was even higher than in Model-1. Apparently, the less accurate SoE estimation of Model-1 led to slower cycle aging. However, Model-2 could yield a lower total cost than Model-1, especially at lower BES prices, because it caused the lowest calendar aging $(1.7 \%)$ and, more importantly, yielded the lowest energy cost (\$4290) out of all models.
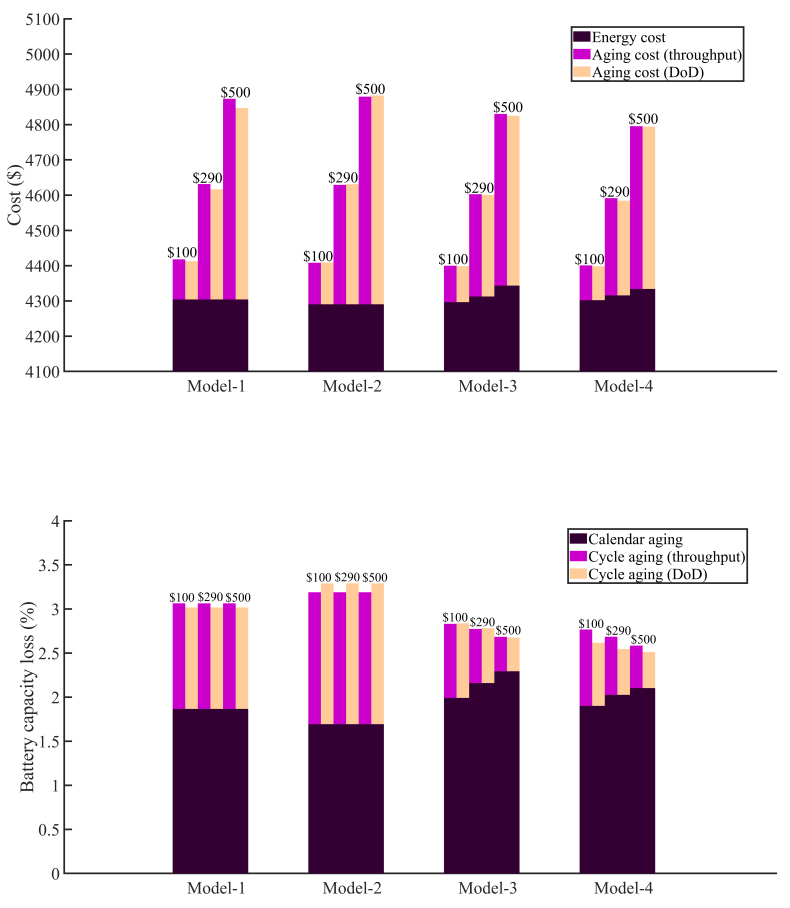

(a) Scenario-1
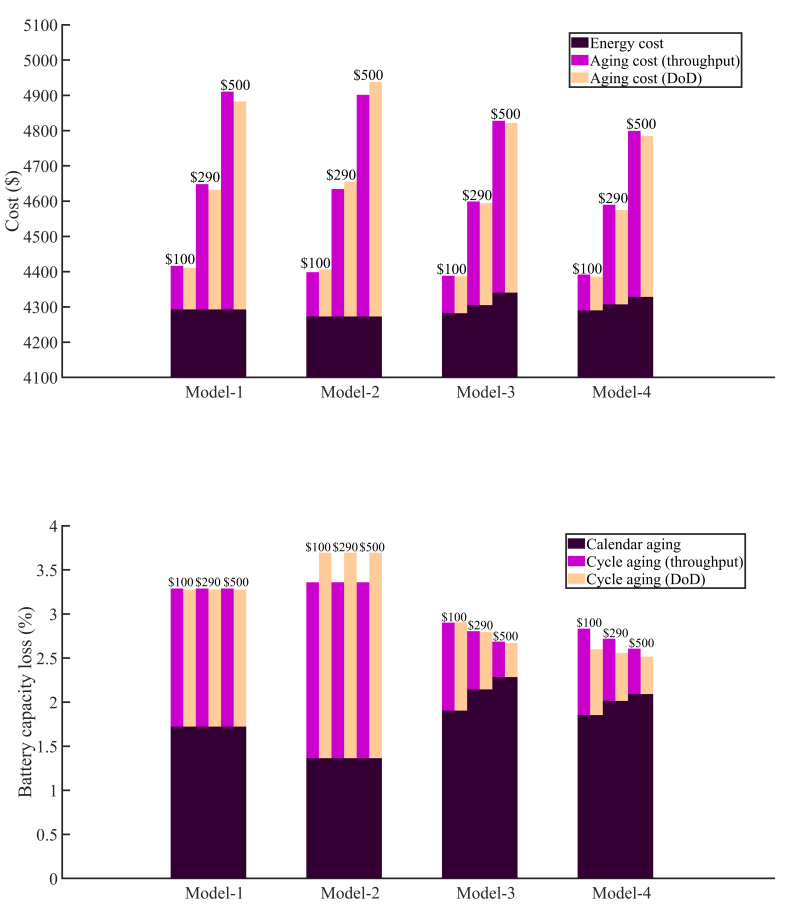

(b) Scenario-2

Fig. 4. The assessment metrics of (a) Scenario-1 (SoE limits of 30\%-80\%) and (b) Scenario-2 (SoE limits of $10 \%-90 \%$ ), where the considered BES installation price is indicated on top of the bars representing the total cost or the total capacity loss. 
2) Scenario-2: Similar trends with Scenario-1 can be observed among the models and across the three BES prices in Scenario-2, Fig. 4(b). The economic performance of Model-3 and Model-4 was equally good in Scenario-2. The total cost of either model was not higher than $\$ 4392$ at \$100. Again, Model-4 yielded the lowest cost at $\$ 290$ and $\$ 500$, which was not higher than $\$ 4590$ and $\$ 4799$, respectively. Apart from the lower energy cost in Scenario-2, the calendar aging also decreased, as lower values of SoE helped the BES retain more capacity during rest periods. This is why these models achieved the best economic performance in Scenario-2 as well.

In contrast, Model-1 and Model-2 gave higher total costs in Scenario-2 at all prices and under both assessments of cycle aging. Again, comparing with the highest cost of either Model1 or Model-2, a reduction of up to $0.7 \%, 1.3 \%$, and $2.3 \%$ could be observed for Model-3 at the price of $\$ 100, \$ 290$, and $\$ 500$, respectively, Similarly, a reduction of up to $0.7 \%, 1.7 \%$, and $3.1 \%$ could be observed for Model-4 at the price of $\$ 100$, $\$ 290$, and \$500, respectively. Model-2 gave the lowest energy cost (\$4273) and the lowest capacity loss due to calendar aging $(1.4 \%)$ out of all models. At the same time, however, it caused the highest cycle-based capacity loss (at least $2 \%$ ) leading to the largest BES degradation, while Model-4 gave the lowest capacity loss in total, which was not higher than $2.8 \%$ at $\$ 100$.

\section{B. Discussion}

The evaluation of Model-3 and Model-4 highlighted the importance of including both energy and degradation cost in the objective functions that determine the BES dispatch.

1) SoE Limits: Model-3 and Model-4 could be used with either conservative or with less strict SoE limits, as both scenarios resulted in almost equal economic benefits for the building owner and the residents. Test with scenarios of different SoE limits are suggested to investigate if the obtained results can be further improved. The choice of optimal SoE limits depends on the possible economic benefit from loadshifting, which is affected e.g., by price fluctuation or PV generation within a day. If the revenue can compensate for the degradation cost, then a larger SoE window can be used.

2) Degradation Models: In both scenarios, Model-4 gave the lowest total cost at $\$ 290$ and $\$ 500$ irrespective of the deviation between the two different assessments of cycle aging. Either Model-3 or Model-4 could achieve the most economic operation at $\$ 100$. Comparing this two models, Model4 caused a lower calendar aging (despite directly forcing the BES to higher SoE values), which contributed to a reduced degradation cost, especially when this was a larger part of the total cost. On the other hand, Model-3 gave lower energy cost, which contributed to the reduction of its total cost, when the degradation cost was a less significant part of it. When cycle aging was omitted from the cost function, there was no clear advantage of using the more accurate measurement-based BES model (Model-2) instead of the conventional one (Model-1).

The accuracy of the aging models can vary depending on the BES chemistry. The aging models used in this paper might not be applicable to all Li-ion BESs. Moreover, it can be hard to obtain reliable aging parameters for each installed stationary BES, if aging models that are derived from empirical fitting are to be used, such as, for example, the cycle aging model presented in Section II-D1 and the calendar aging model.

The cycle aging model presented in Section II-D2, on the other hand, is a more practical modeling approach, as it uses a degradation cost function derived by data provided by the manufacturer, which also adds reliability to the cost of the implemented BES scheduling solution. It should be noted, however, that not all manufacturers provide such detailed data regarding the impact of each cycle to the BES lifetime (often only a maximum number of cycles or throughput is given). Rainflow counting could also be used instead of the half-cycle counting used with this model, however, as explained in [12], the cost function would not be continuously differentiable, and it could therefore not be used in a LP problem formulation. Furthermore, the results (calculated capacity loss after each day-ahead optimization) based on half-cycle counting agreed with the results based on rainflow counting (obtained by the rainflow function in MATLAB [36]), thus validating the halfcycle counting approach presented in Section II-D2.

3) Battery Retirement: The degradation cost in this study is related with $\eta$, which is used to evaluate the remaining useful lifetime of the BES and serves as a termination (retirement) criterion [37]. It is assumed that, when the BES capacity is reduced to $\eta \%$ of its initial value, the BES is replaced. However, storing electricity generated from RES is a less demanding function than powering electric vehicles. Stationary BESs with reduced capacity could still be used in energy management, which could potentially lead to an overestimation of the degradation cost, if the BES is ultimately replaced at a capacity, which is lower than $\eta$. In practice, the BES needs to be replaced after a certain part of its initial capacity is lost, as the overall BES performance deteriorates. The choice of $\eta$, however, is still an open question, as there are relatively few research studies and applications of residential, stationary BES.

4) Revenue Streams: The motivation for focusing on energy arbitrage as the main revenue stream is twofold: 1) operational policies that reduce degradation have a more severe impact on the profit from energy arbitrage than the profit from balancing services [38], which makes the contribution of the measurement-based model in reducing the energy cost all the more important, and 2) there are strong indications that energy arbitrage would be the preferred revenue stream for residential BES owners, who consider protection against high electricity prices as one of the main motives for installation of residential BESs [39]. The proposed model could also be extended to study other revenue streams. Participation of residential BESs in frequency regulation would be an interesting topic for future research, although it should be noted that frequency regulation is more practical at the aggregated level, due to minimum capacity requirements [8] and because it depends on signals from the grid operator [40], which would complicate the control of multiple small-scale BESs. 


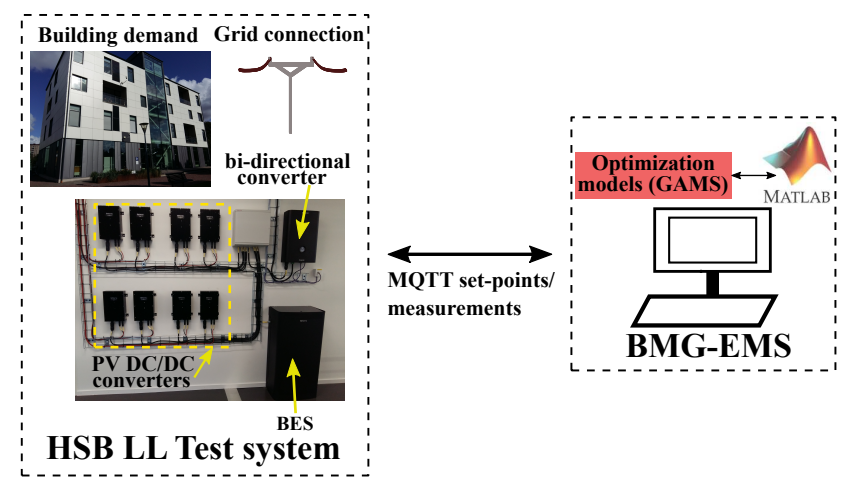

Fig. 5. Control and communication platform of the BMG-EMS.

\section{Demonstration Results}

A BMG-EMS was designed to implement remote control of the BES at the HSB LL building. The BMG-EMS integrated Model-1 and Model-2 and utilized the in-built measurements and control systems of the bi-directional grid side converter.

\section{A. Overview of the Communication and Control Set-up}

The design of the communication platform and the server interfaces can be seen in Fig. 5. The Message Queue Telemetry Transport (MQTT) protocol was used for real-time data sharing between the server, where the BMG-EMS was implemented, and the MQTT broker of the grid side converter. The server interfaced MATLAB [36] to set up the communication and control interface with the test site. A more detailed description of the control set-up can be found in [29]. A MATLAB to GAMS interface was used for data exchange with the optimization models and to retrieve the BES scheduling solution (power set-points), which was dispatched online.

\section{B. Performance Evaluation}

Fig. 6 and Fig. 7 respectively show the BES response during a $24 \mathrm{~h}$ demonstration of Model-1 and Model-2 at HSB LL building. In Fig. 6, it can clearly be observed that the dispatched power did not match the big charging request.

As explained in Section II-C2, SoE mismatches or SoH issues can lead to such deviations from the expected BES performance. This is because the current limiter function of the battery management system is activated to protect the BES from undesired operating conditions, e.g., high temperatures or over/under-voltages (caused by too high/low SoC or degraded BES cells, as was the case here). To mitigate this issue with Model-1 one would have to apply stricter operation limits in upper/lower SoE and BES power. This is, however, a rule-based solution approach that would considerably reduce the BES flexibility. An alternative solution can be found in the measurement-based model. As seen in Fig. 7, the BES response was significantly improved in the demonstration of Model-2 without implementing additional, non-optimal limits. This is because any dependency of the efficiencies and delivered BES power on the SoE that has been captured by the discharging/charging sample data has been considered in the parametrization of this model.

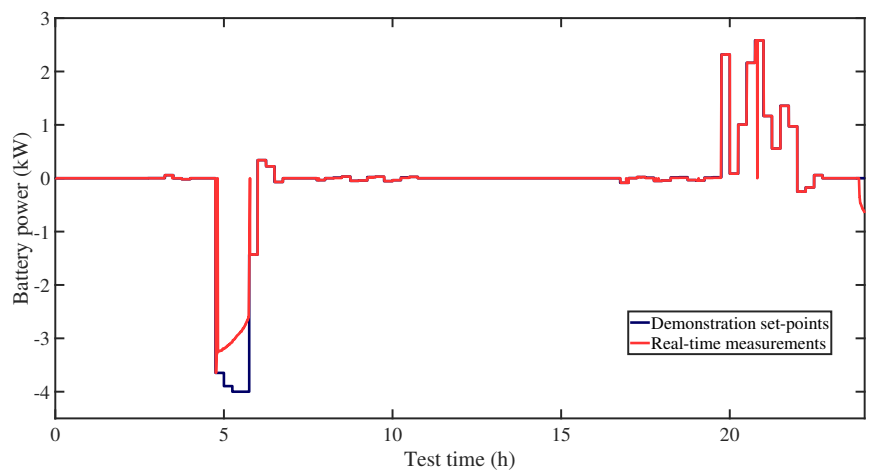

Fig. 6. The set-points and the real-time measurements of the battery power (demonstration of Model-1 at HSB LL building).

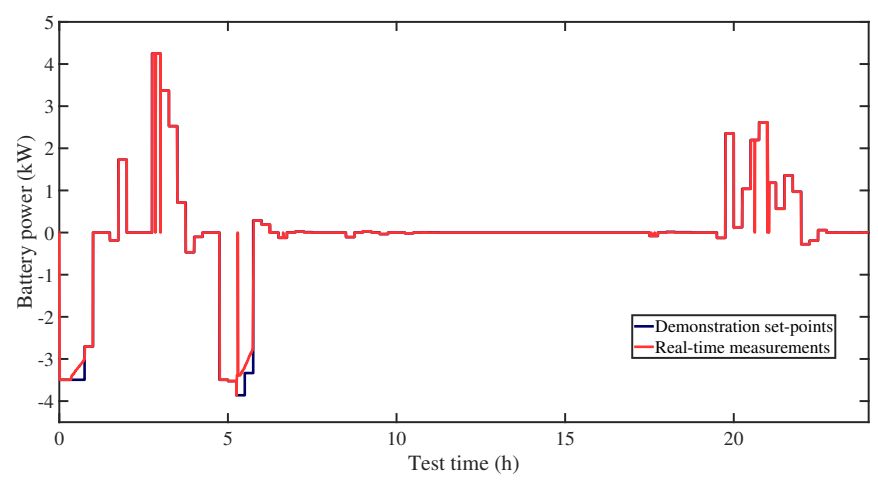

Fig. 7. The set-points and the real-time measurements of the battery power (demonstration of Model-2 at HSB LL building).

The comparison of Model-1 and Model-2 showed that the BES was cycled more when the accuracy of the BES model was improved. This confirms what was indicated by the simulation results, as Model-2 gave a higher cycle aging. Moreover, the total mismatch in delivered charging and discharging BES energy over the requested BES energy was $3.7 \%$ and $13.3 \%$ in the demonstration of Model-2 and Model1 , respectively. This validates the enhanced accuracy of the measurement-based model and, by extent, the reliability of the BES scheduling solution in the simulation results of Section IV, since the measurement-based model was incorporated in Model-2, Model-3, and Model-4 and the assessment of Model1 was performed using the measurement-based model.

\section{CONCLUSIONS}

This paper presents a BMG energy management model that uses BES as a flexible resource considering the energy market-based participation of the buildings. The developed mathematical model incorporates the measurement-based BES model, which can capture realistic performance characteristics, with cycle aging models, while the calendar aging is also calculated after the final dispatch decisions for a comprehensive evaluation of the degradation. The following conclusions can be made from the study performed at the HSB LL, regarding:

- Energy cost and degradation: Model-3 and Model-4, which combined the measurement-based model with cycle aging models dependent on throughput and DoD, 
respectively, could reduce the annual energy and degradation cost by up to $3.1 \%$ compared to when degradation was neglected. The capacity loss was found to be lowest in Model-4. Model-4 also yielded the lowest cost for the BES prices of $\$ 290$ and $\$ 500$.

- SoE limits: It is possible to have more flexible limits on the SoE levels of the BES, if these models are used. On the other hand, if degradation is neglected in the BES dispatch, conservative limits should be applied to prolong the BES lifetime, unless high economic benefits that can offset the degradation cost can be guaranteed.

- Validity of the measurement-based model: A considerable improvement in the BES responses, as compared to those of the conventional BES model, was shown in the real test. As a result, the daily undelivered BES energy over the total request was reduced from $13.3 \%$ to $3.7 \%$ at the same time as the BES usage was increased. This indicates a more reliable implementation of the BES dispatch following the targets of the building operator.

- Potential uses of the model: The proposed model can therefore be employed for both real-time energy management and long-term assessment studies in order to accurately estimate the benefit of BES's flexibilities in reducing the building total costs.

\section{ACKNOWLEDGMENT}

The authors would like to thank Evelina Wikner for fruitful discussions regarding battery degradation and for providing the calendar aging data. Moreover, we would like to thank Ferroamp for providing support with the HSB LL battery.

\section{REFERENCES}

[1] M. C. Argyrou et al., "Energy storage for electricity generation and related processes: Technologies appraisal and grid scale applications," Renewable and Sustainable Energy Reviews, vol. 94, pp. 804-821, Oct. 2018.

[2] S. Dorahaki et al., "Optimal energy management in the smart microgrid considering the electrical energy storage system and the demand-side energy efficiency program," J. of Energy Storage, vol. 28, p. 101229, April 2020.

[3] IRENA, "Electricity storage and renewables: Costs and markets to 2030," Int. Renewable Energy Agency, Abu Dhabi, 2017.

[4] — , "Off-grid renewable energy solutions to expand electricity access: An opportunity not to be missed," Int. Renewable Energy Agency, Abu Dhabi, 2019.

[5] D. Y. Yamashita et al., "A review of hierarchical control for building microgrids," Renewable and Sustainable Energy Reviews, vol. 118, p. 109523, Feb. 2020.

[6] H. Fontenot and B. Dong, "Modeling and control of building-integrated microgrids for optimal energy management-a review," Applied Energy, vol. 254, p. 113689, Nov. 2019.

[7] C. Ju et al., "A two-layer energy management system for microgrids with hybrid energy storage considering degradation costs," IEEE Trans. Smart Grid, vol. 9, no. 6, pp. 6047-6057, May, 2017.

[8] J. E. Contreras-Ocana et al., "Participation of an energy storage aggregator in electricity markets," IEEE Trans. Smart Grid, vol. 10, no. 2, pp. 1171-1183, Mar. 2019.

[9] M. Koller et al., "Defining a degradation cost function for optimal control of a battery energy storage system," in Proc. IEEE Grenoble Conf., Grenoble, France, Nov. 2013.

[10] C. Liu et al., "Economic scheduling model of microgrid considering the lifetime of batteries," IET Gen., Transm. \& Dis., vol. 11, no. 3, pp. 759-767, Feb. 2017.
[11] Y. Shang et al., "Stochastic dispatch of energy storage in microgrids: An augmented reinforcement learning approach," Applied Energy, vol. 261, p. 114423, Mar. 2020.

[12] Y. Shi et al., "A convex cycle-based degradation model for battery energy storage planning and operation," in Proc. Annual American Control Conference (ACC), Wisconsin, US, Aug. 2018, pp. 4590-4596.

[13] I. Alsaidan et al., "A comprehensive battery energy storage optimal sizing model for microgrid applications," IEEE Trans. Power Syst., vol. 33, no. 4, pp. 3968-3980, July 2018.

[14] J. A. Pinzon et al., "Optimal management of energy consumption and comfort for smart buildings operating in a microgrid," IEEE Trans. Smart Grid, vol. 10, no. 3, pp. 3236-3247, May 2019.

[15] G. Cardoso et al., "Battery aging in multi-energy microgrid design using mixed integer linear programming," Applied Energy, vol. 231, pp. 10591069, Dec. 2018.

[16] P. Zhuang and H. Liang, "Hierarchical and decentralized stochastic energy management for smart distribution systems with high BESS penetration," IEEE Trans. Smart Grid, vol. 10, no. 6, pp. 6516-6527, Nov. 2019.

[17] H. Pandžić and V. Bobanac, "An accurate charging model of battery energy storage," IEEE Trans. Power Syst., vol. 34, no. 2, pp. 14161426, Mar. 2019.

[18] A. J. Gonzalez-Castellanos et al., "Non-ideal linear operation model for li-ion batteries," IEEE Trans. Power Syst., vol. 35, no. 1, pp. 672-682, Jan. 2020.

[19] H. Farzin et al., "A stochastic multi-objective framework for optimal scheduling of energy storage systems in microgrids," IEEE Trans. Smart Grid, vol. 8, no. 1, pp. 117-127, Jan. 2017.

[20] M. Sedighizadeh et al., "Stochastic multi-objective economicenvironmental energy and reserve scheduling of microgrids considering battery energy storage system," Int. J. of Elect. Power \& Energy Syst., vol. 106, pp. 1-16, Mar. 2019.

[21] J. Schmalstieg et al., "From accelerated aging tests to a lifetime prediction model: Analyzing lithium-ion batteries," in Proc. IEEE World Electric Vehicle Symp. and Exhib. (EVS27), Barcelona, Spain, Nov. 2013.

[22] E. Wikner and T. Thiringer, "Extending battery lifetime by avoiding high SOC," Applied Sciences, vol. 8, no. 10, p. 1825, Oct. 2018.

[23] J. Wang et al., "Degradation of lithium ion batteries employing graphite negatives and nickel-cobalt-manganese oxide+ spinel manganese oxide positives: Part 1, aging mechanisms and life estimation," J. of Power Sources, vol. 269, pp. 937-948, Dec. 2014.

[24] D. Wang et al., "Quantifying electric vehicle battery degradation from driving vs. vehicle-to-grid services," J. of Power Sources, vol. 332, pp. 193-203, Nov. 2016

[25] M. A. Ortega-Vazquez, "Optimal scheduling of electric vehicle charging and vehicle-to-grid services at household level including battery degradation and price uncertainty," IET Gen., Transm. \& Dis., vol. 8, no. 6, pp. 1007-1016, June 2014.

[26] GAMS. [Online]. Available: https://www.gams.com/

[27] CPLEX https://www.ibm.com/analytics/cplex-optimizer

[28] HSB, "HSB living lab." [Online]. Available: https://www.hsb.se/hsblivinglab/

[29] K. Antoniadou-Plytaria et al., "Chalmers campus as a testbed for intelligent grids and local energy systems," in 2019 Int. Conf. on Smart Energy Systems and Technologies (SEST), Porto, Portugal, Sep. 2019.

[30] Nord Pool. [Online]. Available: https://www.nordpoolgroup.com/

[31] Göteborg Energi. [Online]. Available: https://www.goteborgenergi.se/

[32] J. Ma and X. Ma, "A review of forecasting algorithms and energy management strategies for microgrids," Syst. Sci. \& Control Eng., vol. 6, no. 1, pp. 237-248, June 2018.

[33] R. Weron, "Electricity price forecasting: A review of the state-of-the-art with a look into the future," Int. J. of Forecasting, vol. 30, no. 4, pp. 1030-1081, Oct.-Dec. 2014.

[34] M. Henke and G. Hailu, "Thermal management of stationary battery systems: A literature review," Energies, vol. 13, no. 16, p. 4194, Aug. 2020.

[35] "IEEE/ASHRAE Guide for the Ventilation and Thermal Management of Batteries for Stationary Applications, year=2012, volume=, number=, pages=1-108," IEEE Std 1635-2012/ASHRAE Guideline 21-2012.

[36] MATLAB 2018b, The MathWorks, Inc., Natick, Massachusetts, United States. [Online]. Available: https://www.mathworks.com/ 
[37] X. Li et al., "Multi-time-scale framework for prognostic health condition of lithium battery using modified Gaussian process regression and nonlinear regression," vol. 467, p. 228358, Aug. 2020.

[38] A. Perez et al., "Effect of battery degradation on multi-service portfolios of energy storage," IEEE Trans. Sust. Energy, vol. 7, no. 4, pp. 17181729, Oct. 2016.

[39] B. J. Kalkbrenner, "Residential vs. community battery storage systemsConsumer preferences in Germany," Energy Policy, vol. 129, pp. 1355 1363, June 2019.

[40] B. Cheng and W. B. Powell, "Co-optimizing battery storage for the frequency regulation and energy arbitrage using multi-scale dynamic programming," IEEE Trans. Smart Grid, vol. 9, no. 3, pp. 1997-2005, May 2018.

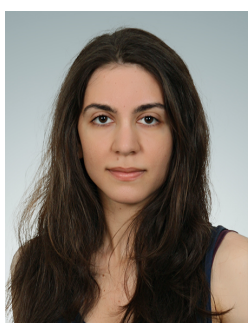

Kyriaki Antoniadou-Plytaria (Graduate Student Member, IEEE) received the diploma in Electrical and Computer Engineering from the National Technical University of Athens (NTUA), Greece, in 2016. Currently, she is pursuing the Ph.D. degree at Chalmers University of Technology, Gothenburg, Sweden. Her research interests are the modeling and optimization of the smart grid operation and microgrid energy management considering integration of distributed energy resources.

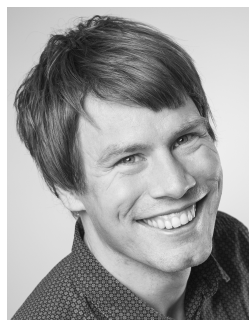

David Steen received the M.Sc. and Ph. D. degrees in electrical engineering from the Chalmers University of Technology in 2008 and 2014, respectively. Currently, he is a researcher in the Department of Electrical engineering, Chalmers University of Technology, Gothenburg, Sweden. His research interests include modeling and control of integrated energy systems and distributed energy resources such as solar PV, wind power, electric vehicles, energy storages.

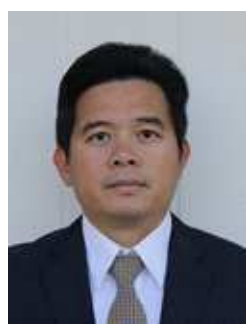

Le Anh Tuan (Member, IEEE) received the BSc degree in power systems from Hanoi University of Technology, Vietnam in 1995, the MSc degree in energy economics from the Asian Institute of Technology, Bangkok, Thailand, in 1997, and the $\mathrm{PhD}$ degree in power systems from the Chalmers University of Technology, Gothenburg, Sweden, in 2004. He is currently a Senior Lecturer with the Division of Electric Power Engineering, Department of Energy and Environment, Chalmers University of Technology. His current research interests include: Modelling, optimization, control and protection of integrated energy systems and active distribution networks with high level of renewables and energy storages; Wide-area monitoring and control of large power transmission systems; Machine learning applications to power systems; Modelling and design of energy and ancillary service markets.

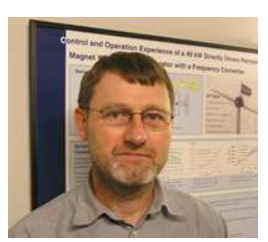

Ola Carlson was born in Onsala, Sweden 1955. He received the M.Sc. and Ph.D. degree in Electrical Engineering from Chalmers University of Technology, Gothenburg, Sweden in 1980 and 1988, respectively. He is currently a Professor at the Department of Electrical Engineering at Chalmers University of Technology. His major interests are electrical systems for renewable power production and hybrid electric vehicles. Since 2010, Ola is the Director of Swedish Wind Power Technology Centre.

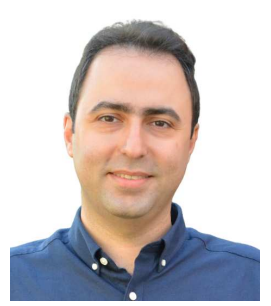

M. Ali Fotouhi Ghazvini (Member, IEEE) received M.Sc. and Ph.D. degrees in electrical engineering from K. N. Toosi University of Technology, Iran and the University of Lisbon, Portugal in 2009 and 2018, respectively. He is currently a researcher at the department of electrical engineering, Chalmers University of Technology, Gothenburg, Sweden. His research interests include operation and control of integrated multi-energy systems, energy management systems, and power system scheduling. 